\title{
Reduced graphene oxide contains a minimum of six oxygen atoms for higher dipolar strength: A DFT study
}

\author{
Narinder Kumar, Bhavna Pal, Shivani Chaudhary, Devendra Singh*, Devesh Kumar* \\ Department of Physics, School for Physical \& Decision Sciences, Babasaheb Bhimrao Ambedkar \\ University, Vidya Vihar, Raebareli Road, Lucknow (U.P.) 226025 INDIA
}

\section{devendras193@gmail.com,dkclcre@yahoo.com}

Keywords: Reduced graphene oxide, Doping of oxygen atom, DFT (B3LYP), Bandgap, Magnetic properties.

The present work focused on the reduced graphene oxide contains a minimum of six oxygen atoms for the higher dipolar strength. The ionization potential and electron affinity decreased only for the six oxygen atoms based graphene. The six oxygen atoms based graphene have the highest dipole moment. The reduced graphene has $0.25 \mathrm{eV}$ bandgap, which is very suitable for electron transfer. The six oxygen atoms based graphene leads to the least gauge including atomic orbital (GIAO) rotational tensor; however, it has the highest isotropic polarizability difference, diamagnetic susceptibility tensor difference, paramagnetic susceptibility tensor difference, and total susceptibility. The $\mathrm{C}-\mathrm{C}$ bond length has increased only for the six oxygen atoms based graphene.

\section{Introduction}

Graphene oxide is a type of nanomaterial which is water-soluble. It has prepared by the chemical action of oxygen with graphite crystals. The sheets of graphene oxide are planar in structure. For enhanced mechanical properties and excellent electrical conductivities, graphene oxide sheets are used for the different applications [1]. The oxygen content in the graphene oxide monolayers is found in a vast amount. Layers of graphene oxide are used in the field-effect transistor. In the manufacturing of biosensor, graphene oxide is helpful. For lightemitting diode and solar cell devices, a visible transparent electrode is required, which can be achieved through the use of graphene oxide [2]. In the graphene oxide, oxygen atoms are connected with graphene along basal edges and corners. The conductive nature of graphene oxide depends on its chemical and atomic structure. Here, the type of hybridization plays a significant role. The hybridization makes it a classical carrier transport material [3]. By adopting different methods of reduction like chemical and thermal, the resistance of graphene material 
could be reduced up to several orders. Now, this graphene oxide will become a semi-metal like graphene. The theoretical study reveals that the bandgap varies up to certain electron volts [4]. Due to the hydrophilic nature of graphene oxide, it is capable of forming aqueous colloids; this helps in large scale use of graphene. For the change in physical properties of Graphene oxide to graphene, the removal of functional groups is necessary, and an insulating material will thus get converted into semi metal. With respect to water solubility, graphene is hydrophobic [5]. With the help of the thin-film technique, graphene oxide is easily deposited in electronic devices. In the biomedical field, graphene oxide is used as a component in the drug delivery system. Just as carbon nanotube (CNT), reduced graphene oxide can also be used as a microwave absorbing material and also as a shielding material for electromagnetic interface [6]. With the increase in frequency, the value of permittivity of reduced graphene oxide decreases; this shows that the dielectric response of reduced graphene oxide is frequencydependent [7]. The conductivity of reduced graphene oxide is lesser than that of graphite because of the reduction process, which shows the presence of residual groups [8]. For microwave absorption, an impedance match characteristic is essential; however, a tremendous value of permittivity is risky for impedance matching. As a result, strong reflection and weak absorption take place in graphite; therefore, reduced graphene is a better option [9]. It also has a disadvantage in the form of dielectric loss and magnetic loss. The novel materials, such as carbon nanotubes and graphene, are difficult to be used in an integrated form [10]. In the present work, we are introducing that reduced graphene contains a minimum of six oxygen atoms for the higher dipolar strength, which is more suitable for the anisotropy and electrical conductivity. The reduced graphene has more exceptional applications as compared with pure graphene; secondly, the pure graphene is costly; however, reduced graphene is cheap and easy to manufacture. The motivation of this work is we can predict doping of other unknown organic materials with pure graphene.

\section{Computational methodology}

All the molecules are optimized by the Gaussian 09 Software package [11] with the help of density functional theory (DFT) method B3LYP [12-13] by $6-31 \mathrm{G}^{* *}$ basis set [14]. With the help of python aggregation [15], the oxygen atoms are doped in the graphene and optimized 300 number of times and found the most stable conformation. The oxygen atoms are step by step added in the graphene and optimized by DFT methodology, as shown in Figures 1 and 2. Here, minimum negative energy-based conformations are concerning because of minimum energy based conformations are more stable for the reaction mechanism and other purposes. The B3LYP function and $6-31 \mathrm{G}^{* *}$ basis set are suitable for the organic compound; however, in the case of 
metals, this strategy not useful, thereby another DFT methodology (PBE) and the basis set (def2tzvp) is helpful.

\section{Results and Discussion}

Six oxygen atoms based graphene having more significant absorbance due to out of plane wagging of $\mathrm{OH}$ atom as well as $\mathrm{C}-\mathrm{C}$ atom stretching, thereby reduced graphene increases the bond length. Six oxygen atoms based graphene also express rocking and stretching of $\mathrm{OH}$ atom, which enhances the absorbance of the reduced graphene. However, five and seven atoms based graphene have only revealed the rocking and scissoring of $\mathrm{OH}$ atom and therefore express the lower absorbance as a comparison with six atoms based graphene. Khoei et al. [16] have reported the $\mathrm{C}-\mathrm{C}$ atom bond length of each hexagonal lattice increases due to $\mathrm{OH}$ group doped in the graphene; the present work also expresses the bond length increases only for the six oxygen atoms based graphene as shown in Figure 2 and given in Table 3. Leenaerts et al. [17] have reported the doping of water cluster with graphene-enhanced the dipole moment and expressed very small resistance in the present work also the dipole moment increased only for the 6th oxygen atom and expressed $0.2 \mathrm{eV}$ bandgap which is suitable for the electron transfer from highest occupied molecular orbital (HOMO) to lowest unoccupied molecular orbital (LUMO) as given in Table 1. Hernandez et al. [18] have reported DFT as the most suitable tool for the prediction of graphene and water molecule interaction in the present work also, DFT(B3LYP) methodology is used for the experimental evidence. Abid et al. [19] have reported the increased doping of oxygen atom in the graphene to enhance the bandgap of graphene oxide here; we found that bandgap increased with doping of oxygen atom so we can say theoretical prediction correlated with the experimental evidence as given in Table 1. Yang et al. [20] have reported the polarity of graphene oxide connected with the adsorption ability to different monomers, and these monomers modify the capability of the graphene oxide for the different applications. Acik et al. [21] have reported in the review doping of oxygen atom tune the bandgap of graphene oxide. By the $\mathrm{Ab}$ initio simulation, it has been found that the $11.1 \%$ ratio of $\mathrm{O} / \mathrm{C}$ (oxygen/carbon) expresses the $0.35 \mathrm{eV}$ bandgap. Here 6th, oxygen atom gives the $0.25 \mathrm{eV}$ bandgap, which has correlated with the simulation prediction. The bandgap of reduced graphene oxide exists between $2.8 \mathrm{eV}$ to $0.02 \mathrm{eV}$; Here, reported bandgap is existing between in this range. Mathkar et al. [22] have reported gasbased hydrazine strategy is more suitable rather than the other synthesis methods for the preparation of reduced graphene oxide. Miao et al. [23] have reported an increased amount of nitrogen atom doping in graphene to enhance the magnetic properties in the present nitrogen atom replaced with an oxygen atom, which also improves the magnetic properties of reduced 
graphene oxide as given in Table 2. Singh et al. [24] have reported six oxygen atoms based reduced graphene oxide interacted with the polymer (7OBA) in $\pi-\pi$ stacking and enhanced the electric conductivity as well as dielectric anisotropy of the composite which is more suitable for the nonlinear applications. The dipolar strength has increased for the six oxygen atoms, as shown in Figure 3 and expressed in Table 1. The B3LYP function with the $6-31 \mathrm{G}^{* *}$ basis set is most suitable for the organic compound that is the reason for choosing this strategy [24-25].

Table 1. Description of the gauge including atomic orbital (GIAO) rotational tensor, dipole moment, bandgap and total energy of reduced graphene oxide with an expansion of oxygen atom

\begin{tabular}{|c|c|c|c|c|}
\hline $\begin{array}{c}\text { No. of } \\
\text { Oxygen } \\
\text { atoms }\end{array}$ & $\begin{array}{c}\text { GIAO } \\
\text { (a.u.) }\end{array}$ & $\begin{array}{c}\text { Dipole } \\
\text { Moment } \\
\text { (Debye) }\end{array}$ & $\begin{array}{c}\text { Bandgap } \\
(\mathrm{eV})\end{array}$ & $\begin{array}{c}\text { Energy } \\
\text { (a.u.) }\end{array}$ \\
\hline 1 & -0.0019 & 2.95 & 0.25 & -2833.71 \\
\hline 2 & -0.0018 & 6.46 & 0.25 & -2908.93 \\
\hline 3 & -0.0017 & 5.73 & 0.25 & -2984.15 \\
\hline 4 & -0.0016 & 5.57 & 0.25 & -3059.37 \\
\hline 5 & -0.0017 & 5.97 & 0.26 & -3134.58 \\
\hline $\mathbf{6}$ & $\mathbf{- 0 . 0 0 1 5}$ & $\mathbf{7 . 5 6}$ & $\mathbf{0 . 2 5}$ & $\mathbf{- 3 2 0 9 . 8 0}$ \\
\hline 7 & -0.0018 & 5.35 & 0.27 & -3285.02 \\
\hline 8 & -0.0019 & 7.10 & 0.27 & -3360.25 \\
\hline
\end{tabular}

Table 2. Description of the diamagnetic susceptibility, paramagnetic susceptibility and total susceptibility with an expansion of oxygen atom

\begin{tabular}{|c|l|l|l|}
\hline $\begin{array}{l}\text { No. of } \\
\text { Oxygen } \\
\text { atoms }\end{array}$ & $\begin{array}{l}\text { Diamagnetic } \\
\text { susceptibility } \\
\text { (a.u.) }\end{array}$ & $\begin{array}{l}\text { Paramagnetic } \\
\text { susceptibility } \\
\text { (a.u.) }\end{array}$ & $\begin{array}{l}\text { Total } \\
\text { susceptibility } \\
\text { (a.u.) }\end{array}$ \\
\hline 1 & -10401.51 & 10251.51 & -149.99 \\
\hline 2 & -10863.84 & 10714.50 & -149.33 \\
\hline 3 & -11355.02 & 11203.70 & -151.31 \\
\hline 4 & -11851.87 & 11698.74 & -153.13 \\
\hline 5 & -12138.48 & 11986.00 & -152.48 \\
\hline $\mathbf{6}$ & $\mathbf{- 1 2 6 0 7 . 7 3}$ & $\mathbf{1 2 4 5 4 . 8 3}$ & $\mathbf{- 1 5 2 . 8 9}$ \\
\hline 7 & -12888.30 & 12736.14 & -152.15 \\
\hline 8 & -13278.63 & 13129.01 & -149.62 \\
\hline
\end{tabular}

Table 3. Description of C-C bond length of the benzene ring in the reduced graphene oxide with 5, 6 and 7 oxygen atoms

\begin{tabular}{|c|c|c|c|}
\hline $\begin{array}{l}\text { Number of carbon } \\
\text { atoms }\end{array}$ & $\begin{array}{l}\text { C-C } \\
\text { bond } \\
\text { length } \\
\text { with } 5 \\
\text { oxygen } \\
\text { atoms }\end{array}$ & $\begin{array}{l}\text { C-C } \\
\text { bond } \\
\text { length } \\
\text { with } 6 \\
\text { oxygen } \\
\text { atoms }\end{array}$ & $\begin{array}{l}\text { C-C } \\
\text { bond } \\
\text { length } \\
\text { with } 7 \\
\text { oxygen } \\
\text { atoms }\end{array}$ \\
\hline C73-C18 & 1.42310 & 1.42303 & 1.42258 \\
\hline C18-C21 & 1.43059 & 1.43068 & 1.43029 \\
\hline C21-C65 & 1.42413 & 1.42419 & 1.42348 \\
\hline C65-C68 & 1.42301 & 1.42303 & 1.42352 \\
\hline C68-C70 & 1.43037 & 1.43048 & 1.43004 \\
\hline C70-C73 & 1.42268 & 1.42274 & 1.42220 \\
\hline
\end{tabular}




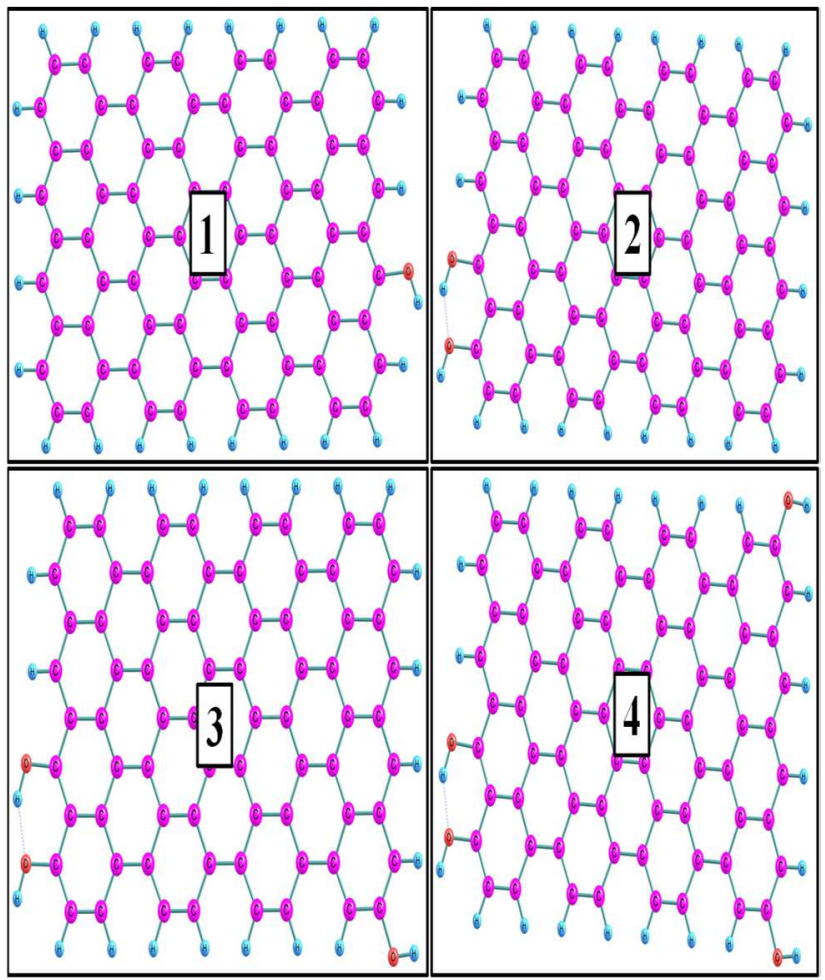

Figure 1. Step by step optimized reduced graphene oxide with an extension of oxygen atom by random position and more stable conformation.

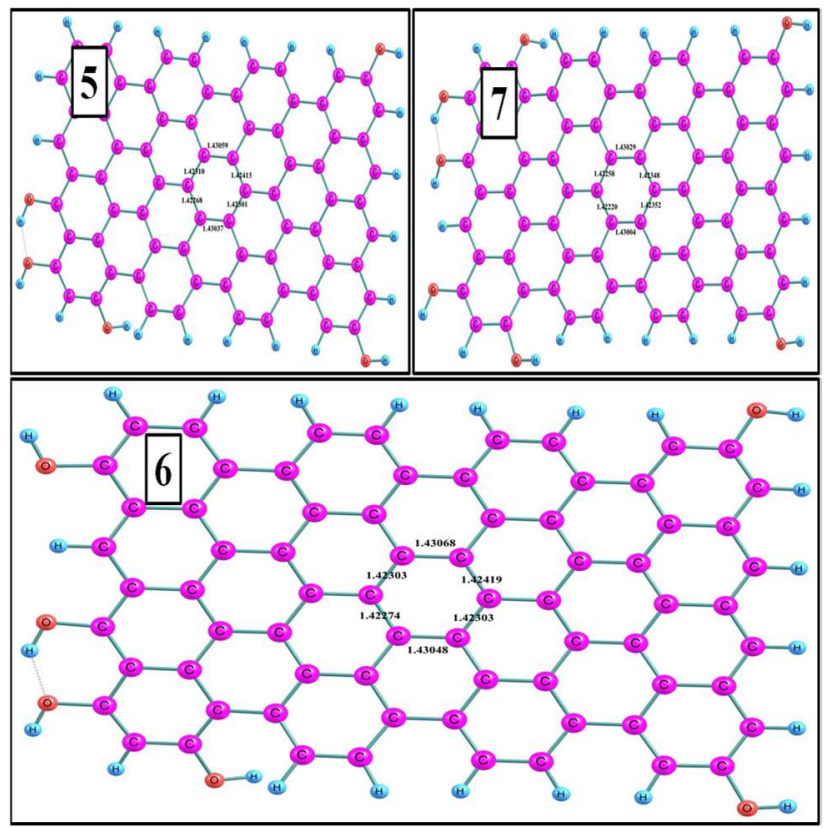

Figure 2. The optimized geometry of five, six and seven atoms based reduced graphene oxide indicating $\mathrm{C}-\mathrm{C}$ atom bond length, six oxygen atoms based graphene expresses a maximum change in bond length

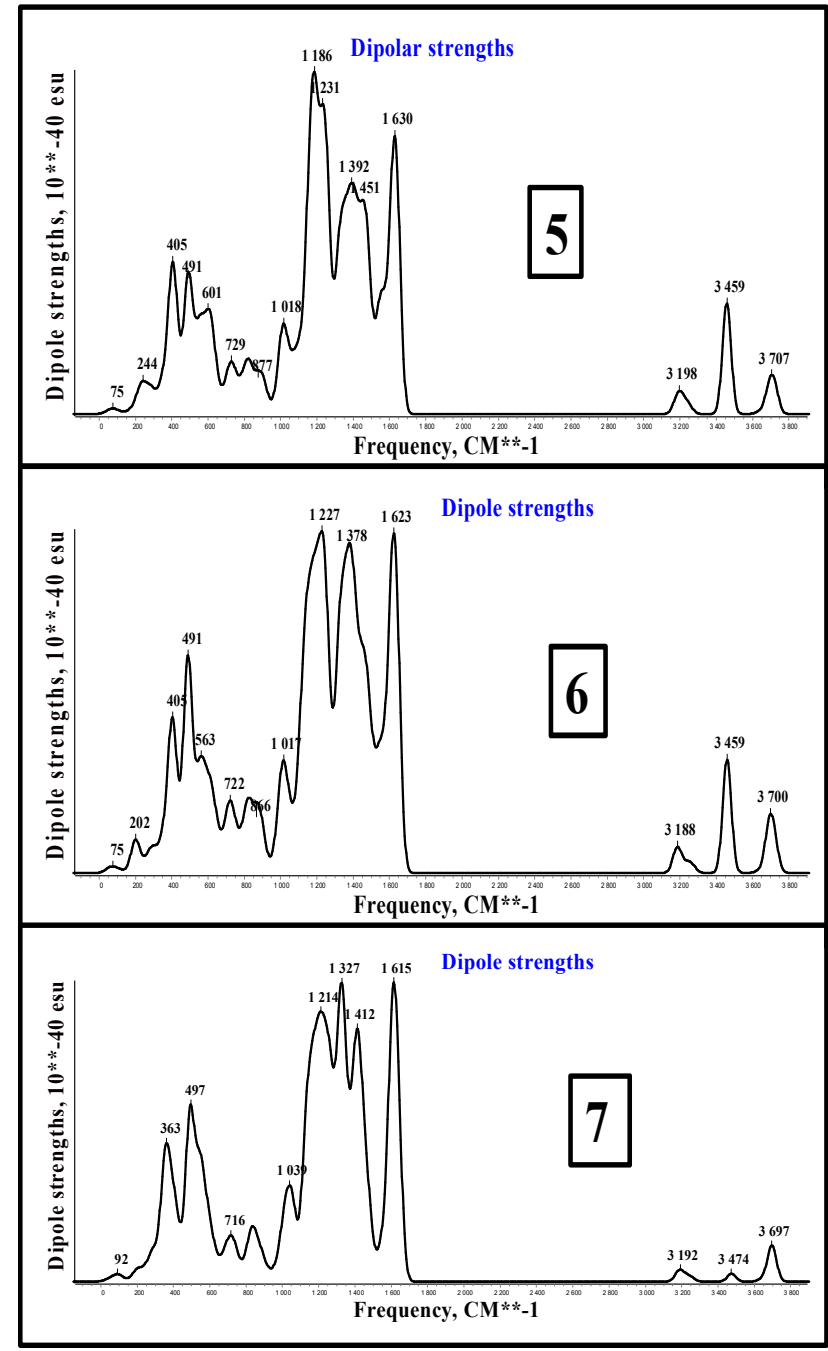

Figure 3. Dipolar strength of five, six, and seven oxygen atoms based on reduced graphene oxide. The six oxygen atoms based on reduced graphene, indicating more dipolar strength.

\section{Conclusions}

In the present work, it has been found that reduced graphene oxide contains a minimum of six oxygen atoms for higher dipolar strength. The $\mathrm{C}-\mathrm{C}$ atoms bond length and dipole moment have enhanced only for the six oxygen atoms based graphene. The ionization potential and electron affinity reduced only for six oxygen atoms based graphene. The six oxygen atoms based graphene oxide have the least GIAO rotational tensor; 
however, it has the highest isotropic polarizability difference, diamagnetic susceptibility tensor difference, paramagnetic susceptibility tensor difference, and total susceptibility. Graphene oxide has a high surface area, so it is more suitable for use as electrode material for batteries, solar cells, chemical sensors, and capacitors. Graphene oxide is cheaper and easier to manufacture as compared with pure graphene. The mixture of polymer and graphene oxide enhances the properties of the complex like elasticity, tensile strength, conductivity, ion conductors, hydrogen storage, and nanofiltration membranes. The six oxygen atoms based graphene expressed the $\pi$ - $\pi$ stacking with the liquid crystal materials, which is suitable for the solar cell and other application devices.

\section{Acknowledgment}

N. Kumar is thankful to the University Grants Commission (UGC) New Delhi for providing fellowship (RGNF) for this work. We are very grateful to Dr. A. Anoop Ayyappan, Associate Professor in the department of chemistry (IIT Kharagpur, West Bengal), for providing computational help for this work. Ms. S. Chaudhary and Ms. Bhavna Pal are thankful to UGC for providing a non-NET fellowship.

\section{References}

[1] Hidayah, NMS, Liu, W, Lai, C, Noriman, N, Khe, C, Hashim, U, Lee, HC. “Comparison on graphite, graphene oxide and reduced graphene oxide:
Synthesis and Characterization” AIP Conf. Proc. 2017; 1892:1500021-1500028.

[2] Kumar, CV, Pattammattel, A. "Introduction to graphene:Chemical and biological applications" Elsevier 2017.

[3] Pisula, W, Feng, X, Mullen, K. Charge carrier transporting graphene type molecules Chem. Mater. 2011; 23:554-567.

[4] Warner, JH, Schaffel, F, Rummeli, M, Bachmatiuk, A. Graphene: Fundamentals and emergent applications Technology \& Engineering, 2012.

[5] Shafraniuk, S. Thermoelectricity and heat transport in Graphene and other 2D Nanomaterials Elsevier Science, 2017.

[6] Nazarpour, S, Waite, SR. Graphene technology: From laboratory to fabrication John Wiley \& Sons, Technology \& Engineering, 2016.

[7] Torres, T. Carbon nanotubes and related structures: Synthesis, characterization, functionalization and applications Angew. Chem. Int. Ed. 2011;50:1473-1474.

[8] Rowley-Neale, SJ, Randvirr, J, Abo Dena, EP. An overview of recent applications of reduced graphene oxide as a basis of electroanalytical sensing platforms Applied materials today, 2018;10:218-226.

[9] Ahmad, H, Fan, M, Hui, D. Graphene oxide incorporated functional materials: A review Composites part B: Engineering, 2018;145:270280.

[10]Geim, AK. Graphene Prehistory Physica Scripta, 2012;T146:014003-014007.

[11]Frisch, MJ Trucks, GW, Schlegel, HB, . Scuseria, GE, Robb, MA. et al. Gaussian 09, Revision A.02, Gaussian, Inc., Wallingford CT, 2010.

[12]Becke, AD. Density-functional thermochemistry. III. The role of exact exchange, J. Chem. Phys. 1993;98:5648-5652. 
[13]Kumar, N, Singh, P, Upadhyay, P, Chaudhary, S, Thapa, KB, Dwivedi, AK, Kumar, D. Odd-even effect of 7O.m liquid crystal compound series studied under the effect of the electric field by density functional theory (DFT) methods, Eur. Phys. J. Plus 2020;135:388.

[14]Petersson, GA, Bennett, A, Tensfeldt, TG, AlLaham, MA, Shirley, WA, Mantzaris, J. A complete basis set model chemistry. I. The total energies of closed-shell atoms and hydrides of the first-row atoms, J. Chem. Phys., 1988;89:21932218.

[15]Khatun, M, Majumdar, RS, Anoop, A. A Global Optimizer for Nanoclusters, Front. Chem. 2019;7:644.

[16]Khoei, AR, Khorrami, MS. Mechanical properties of graphene oxide: A molecular dynamics study, Fullerenes, Nanotubes and Carbon Nanostructures, 2016;24(9):594-603.

[17]Leenaerts, O, Partoens, B, Peeters, FM. Water on graphene: Hydrophobicity and dipole moment using density functional theory, Phys. Rev. B. 2009;79:235440.

[18]Hernandez, M, de Oca, ACM, Oliva-Leyva, M, Naumis, GG. How water makes graphene metallic, Phys. lett. A, 2019;383(29):125904.

[19]Abid, Sehrawat, P, Islam, SS, Mishra, P, Ahmad, S. Reduced graphene oxide (rGO) based wideband optical sensor and the role of Temperature, Defect States and Quantum Efficiency, Sci Rep. 2018; 8: 3537.

[20] Yang, X, Li, J, Wen, T, Ren, X, Huang, Y, Wang, $X$. Adsorption of Naphthalene and Its Derivatives on Magnetic Graphene Composites and the Mechanism Investigation. Colloids Surf., A 2013;422:118-125.

[21]Acik, M, Chabal, YJ, A Review on Thermal Exfoliation of Graphene Oxide, J. Matt. Sci. 2013;2:101-112.
[22] Mathkar, A, Tozier, D, Cox, P, Ong, P, Galande, C, Balakrishnan, K, Reddy, ALM, Ajayan, PM. Controlled, Stepwise Reduction and Band Gap Manipulation of Graphene Oxide. J. Phys. Chem. Lett. 2012;3:986-991.

[23]Miao, Q, Wang, L, Liu, Z. et al. Magnetic properties of $\mathrm{N}$-doped graphene with high Curie temperature. Sci. Rep. 2016;6:21832.

[24] Singh, DP, Misra, AK, Pandey, KK, Pal, B, Kumar, N. et al., Spectroscopic, dielectric and nonlinear current-voltage characterization of a hydrogen-bonded liquid crystalline compound influenced via graphitic nanoflakes: An equilibrium between the experimental and theoretical studies, J. Mol. Liq. 2020;302:112537.

[25]Kumar, N, Singh, P, Upadhyay, P. et al. Oddeven effect of 70.m liquid crystal compound series studied under the effect of the electric field by density functional theory (DFT) methods, Eur. Phys. J. Plus, 2020;135:388.

[26]Kumar, N, Singh, P, Thapa, KB, Kumar, D. Molecular spectroscopy and adverse optical properties of $\mathrm{N}$-(p-hexyloxybenzylidene)-ptoluidine (HBT) liquid crystal molecule studied by DFT methodology, IOP SciNotes, 2020;1:015202. 